\title{
Sodium-glucose co-transporter 2 inhibitors in COVID-19: meeting at the crossroads between heart, diabetes and infectious diseases
}

\author{
Theocharis Koufakis ${ }^{1} \cdot$ Antonis N. Pavlidis $^{2} \cdot$ Symeon Metallidis $^{3} \cdot$ Kalliopi Kotsa $^{1}$ (i)
}

Received: 12 January 2021 / Accepted: 22 February 2021 / Published online: 9 March 2021

(c) The Author(s), under exclusive licence to Springer Nature Switzerland AG 2021

\begin{abstract}
Sodium-glucose co-transporter 2 inhibitors (SGLT2i) are a new class of glucose-lowering agents which have changed the landscape of diabetes therapy, due to their remarkable cardiorenal protective properties. The attack of severe acute respiratory syndrome coronavirus 2 on the heart and kidneys shares similarities with diabetes; therefore, the notion that SGLT2i might have a role in the future management of Coronavirus Disease 2019 (COVID-19) is based on a solid pathophysiological hypothesis. SGLT2 $i$ have been proved to decrease the expression of proinflammatory cytokines, ameliorate oxidative stress and reduce sympathetic activity, thus resulting in downregulation of both systemic and adipose tissue inflammation. On the other hand, they have been linked to an increased risk of euglycemic diabetic ketoacidosis. Therefore, the efficacy and safety of SGLT2i in COVID-19 are still debatable and remain to be clarified by ongoing randomized trials, to assess whether the benefits of treatment with these drugs outweigh the potential risks.
\end{abstract}

Keywords COVID-19 $\cdot$ Diabetes $\cdot$ Organ damage $\cdot$ SGLT2 inhibitors

\section{Background}

Sodium-glucose co-transporter 2 inhibitors (SGLT2i) are a new class of oral, glucose-lowering agents used for the management of type 2 diabetes (T2D), but also as an adjunct treatment in overweight people with type 1 diabetes (T1D), not adequately controlled with insulin. Gliflozins block SGLT2, expressed in the early segment of the proximal renal tube, reducing this way renal glucose reabsorption. This class of drugs has changed the landscape of diabetes therapy, not only because of their potency in lowering blood glucose with a minimal risk of causing hypoglycemia, but

Kalliopi Kotsa

kalmanthou@yahoo.gr

1 Division of Endocrinology and Metabolism and Diabetes Center, First Department of Internal Medicine, Medical School, Aristotle University of Thessaloniki, AHEPA University Hospital, 1 St. Kiriakidi Street, 54636 Thessaloníki, Greece

2 Cardiology Department, St.Thomas' Hospital, London, UK

3 Infectious Diseases Division, First Department of Internal Medicine, Medical School, Aristotle University of Thessaloniki, AHEPA University Hospital, Thessaloníki, Greece predominantly due to their remarkable cardiorenal protective properties. Robust evidence from recently published largescale cardiovascular outcome trials (CVOTs) has associated treatment with SGLT2i with significant reductions in the risk of major cardiovascular (CV) events, hospitalization for heart failure (HF), CV death, and renal outcomes [1,2]. The impressive results of CVOTs, particularly in populations with established atherosclerotic disease, HF, and impaired renal function, have gradually shifted the perspective of diabetes management from a strictly glucose-centric approach to recognizing the importance of addressing $\mathrm{CV}$ risk simultaneously with glycemic control.

During the past months, humanity has been dramatically experiencing the impact of the "Coronavirus Disease 2019 (COVID-19)" pandemic on every aspect of economic and social life, with the disease being a huge burden for health care systems around the world. People with diabetes are known to be at great risk of poor outcomes, including increased mortality rates during COVID-19 [3]. This commentary aims to discuss the pathophysiological background and the risk-benefit balance of the administration of SGLT2i to patients with COVID-19. 


\section{The rationale behind the use of SGLT2i in COVID-19}

It is well established that hyperglycemia exerts unfavorable effects on the CV system through multiple mechanisms, including chronic, low-grade inflammation and endothelial dysfunction that negatively affect the pump function of the heart and result in the development of atherosclerosis [4]. Although the exact mechanisms involved in the heart and kidney benefits of SGLT2i have not been fully elucidated, these are believed to derive from reduction in preload and afterload secondary to natriuresis and osmotic diuresis, changes in heart metabolomics, and downregulation of profibrotic and pro-inflammatory markers [5]. Particularly, they have been shown to suppress leptin, interleukin (IL)-6, and tumor necrosis factor (TNF) $-\alpha$ levels, which are all major contributors to the development of cardiac inflammation [6]. SGLT2i have been proved to ameliorate oxidative stress, reduce sympathetic activity and improve cell autophagy, thus, resulting in downregulation of both systemic and adipose tissue inflammation [7]. Canagliflozin and dapagliflozin have been demonstrated to activate the AMP-activated protein kinase, resulting in reduced inflammatory responses in endothelial cells and myofibroblasts, respectively [5-7].

Interestingly, the attack of severe acute respiratory syndrome coronavirus 2 (SARS-CoV-2) on the heart seems to share similarities with diabetes pathogenetic pathways. Cytokine storm, direct viral damage and endotheliitis are all believed to be implicated in the cardiac manifestations of the disease, such as myocarditis, HF, and myocardial infarction. From a clinical perspective, the incidence of myocardial damage in COVID-19 patients has been estimated to be as high as $28 \%$, related to poor prognosis [8]. Cardiac arrhythmias are more prevalent among critically ill patients with COVID-19, related not only to direct viral effects, but also to systemic inflammation and drug interactions [9]. Recent data suggest that SGLT2i might confer protection against newonset arrhythmias in people with T2D [adjusted hazard ratio (aHR) 0.83], with this benefit also translated into a lower risk of all-cause mortality (aHR 0.55) [10]. It is worth mentioning that relevant data are mainly driven by a significant reduction in the risk for atrial fibrillation $(\mathrm{AF})$ observed with treatment with dapagliflozin in high-risk individuals with $\mathrm{T} 2 \mathrm{D}$, regardless of the patient's former history of AF, CV disease or HF [11]. Although the exact mechanisms mediating the antiarrhythmic effects of SGLT2i are still obscure, it has been postulated that these are driven by structural, electromechanical, and mechanical myocardial remodeling and downregulation of the sympathetic tone $[5,10,11]$.

Lactate dehydrogenase concentrations positively correlate with tissue hypoxia and have been suggested to reflect the severity of organ damage and relate to increased mortality rates in COVID-19 [12]. Normally, $\mathrm{Na}^{+} / \mathrm{H}^{+}$exchanger (NHE), along with $\mathrm{H}^{+} /$lactate symporter, contribute to the regulation of intracellular $\mathrm{PH}$. Increase of lactate in the extracellular space during hypoxia activates the symporter which moves lactate and $\mathrm{H}^{+}$into the cell but also the NHE, which exports $\mathrm{H}^{+}$and imports $\mathrm{Na}^{+}$and $\mathrm{Ca}^{++}$, leading to cell edema. SGLT2i are known to inhibit NHE flux, resulting in decreased intracellular and mitochondrial $\mathrm{Na}^{+}$and $\mathrm{Ca}^{++}$concentrations, improved myocardial contractility, and preservation of cytosolic PH under hypoxic conditions [7]. In addition, dapagliflozin is believed to inhibit lactate formation mediated by improved oxygen supply to kidneys, while improving at the same time its renal excretion [13].

Endothelial dysfunction, excessive inflammation, ischemic injury and hypercoagulability are considered to be essential mechanisms in the development of acute kidney injury (AKI) in COVID-19. AKI is associated with a 13-fold greater risk of death among SARS-CoV-2 infected individuals [14]. Of note, Menon et al. [15] recently showed that renal damage in diabetes and COVID-19 might share common backgrounds, by demonstrating that the diabetic kidney disease angiotensin converting enzyme 2 (ACE2)positive proximal tubular epithelial cell module overlaps with the expression patterns observed in SARS-CoV-2 infected cells. On the other hand, SGLT2 inhibition results in a natriuretic response, which through tubuloglomerular feedback, causes afferent arteriolar vasoconstriction, finally leading to reductions in intraglomerular pressure. SGLT2i have been shown to increase erythropoietin concentrations leading to improved oxygenation of heart and renal tissue [5]. Moreover, in an experimental model of diabetic kidney disease, canagliflozin decreased plasma concentrations of TNF receptor 1, IL-6, matrix metalloproteinase 7, and fibronectin 1, suggesting that SGLT2i have the potential to reverse molecular processes related to renal inflammation and fibrosis [16].

Based on the above indirect associations, the question then arises: do SGLT2i have the potential to improve COVID-19-related outcomes in people with or even without diabetes? Although the rationale behind such an approach seems valid, the available data are still limited and mainly come from observational studies. In a recently published analysis of the impact of diabetes and hypertension pharmacotherapy on COVID-19 outcomes, Dalan et al. [17] established a lower risk of mechanical ventilation in a small group of patients $(n=16)$ having been exposed to SGLT $2 \mathrm{i}$ treatment, after adjustment for baseline features and CV status. Figure 1 summarizes the putative mechanisms through which SGLT2 inhibitors might alleviate organ damage in COVID-19. 


\section{The other side of the coin}

Safety issues should be considered when discussing the role of these agents in COVID-19. SGLT2i promote a shift of energy substrate usage from carbohydrates to lipids, while decreasing at the same time renal ketone body clearance [5]. Under specific circumstances, including severe infections, emergency surgery, or prolonged fasting, treatment with these drugs might trigger the development of euglycemic diabetic ketoacidosis (DKA), a potentially fatal clinical entity. Armeni et al. [18] have recently reported a profound association between COVID-19 and hyperglycemic emergencies, including DKA and long-lasting ketosis, in people with T2D and COVID-19. In another case series, ChamorroPareja et al. [19] reported that the majority of people presenting with DKA and COVID-19 had T2D and not T1D and contrary to expectation, they experienced good glycemic control before admission. Taking into account the growing number of reports describing patients who presented with euglycemic DKA in the context of COVID-19 and SGLT2i use [20], there is a need for increased vigilance by physicians to properly recognize and treat such cases. The data suggest that SARS-CoV-2 might potentially exert direct diabetogenic effects by attacking beta-cells and significantly impairing insulin secretion, and in conjunction with the exacerbation of insulin resistance due to systemic inflammation, finally leads to relative insulinopenia and the development of DKA [18-20]. Further studies are needed to clarify to what extent co-administration with insulin might alleviate the risk of DKA in people with COVID-19 and T2D receiving SGLT2i.

Another concern is that treatment with SGLT2 $i$ has been linked to upregulation of ACE2 [7], which is the essential SARS-CoV-2 receptor, and might potentially result in increased viral entry into lung and other tissue cells. On the other hand, although women manifest higher ACE2 levels, available evidence suggests significantly higher mortality rates among men [3], indicating that disease severity and progression in COVID-19 is mediated by complex and multiple factors.

\section{Conclusion}

In any case, definite answers regarding the effectiveness and safety of SGLT2i in acute COVID-19 are anticipated from two ongoing, randomized-controlled trials (RCTs): DARE19 (ClinicalTrials.gov, NCT 04350593, $\mathrm{n}=1250$, estimated completion date June 2021) aims to explore whether dapagliflozin could lower the risk of disease progression, complications, and mortality due to COVID-19 in patients with T2D, CV disease, and/or impaired renal function. TACTICE (ClinicalTrials.gov, NCT 04393246, $n=1407$, estimated completion date June 2021) investigates the effects of dapagliflozin when combined with the endothelin receptor agonist ambrisentan, on clinical outcomes (death, mechanical ventilation, $\mathrm{CV}$ organ support, or renal failure) and several biomarkers related to the disease progression (e.g. IL-6, c-reactive protein, d-dimers) in patients with COVID-19.

In conclusion, the notion that SGLT2i might have a role in the future management of COVID-19, although purely speculative for the moment, seems to be based on a solid pathophysiological hypothesis. Efficacy and safety, particularly with respect to the development of DKA, are still debatable and remain to be clarified by ongoing RCTs and accumulation of real-world data, to assess whether the benefits of treatment with these drugs in SARS-CoV-2 infected individuals outweigh the potential risks. In this battle, we have to be both rare and innovative; taking the hard road is sometimes the only option.
Fig. 1 Putative mechanisms through which SGLT2 inhibitors might alleviate organ damage in COVID-19. SGLT2 sodium-glucose co-transporter 2; COVID-19 coronavirus disease 2019; IL-6 interleukin-6; $T N F-\alpha$ tumor necrosis factor- $\alpha$; $M M P 7$ matrix metalloproteinase $7 ; F N 1$ fibronectin $1 ; N H E$ $\mathrm{Na}^{+} / \mathrm{H}^{+}$exchanger

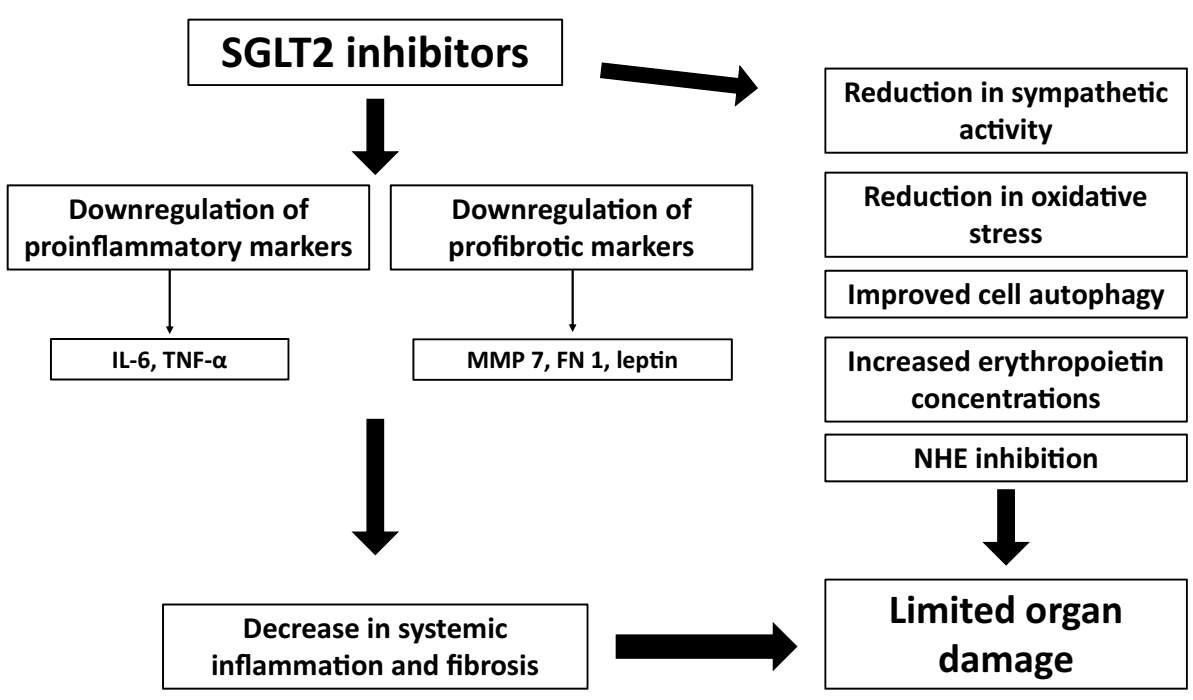


Funding The authors received no financial support for the research, authorship, and/or publication of this article.

Conflicts of interest TK has received honoraria as a speaker from AstraZeneca, Boehringer Ingelheim and Novo Nordisk and has participated in sponsored studies by Eli-Lilly. KK has received honoraria for lectures/advisory boards and research support. from Astra Zeneca, Boehringer Ingelheim, Pharmaserve Lilly, Sanofi-Aventis, ELPEN, MSD, and Novo Nordisk.Other authors report no conflict of interest.

\section{References}

1. Heerspink HJL, Stefánsson BV, Correa-Rotter R, Chertow GM, Greene T, Hou FF, et al. Dapagliflozin in patients with chronic kidney disease. N Engl J Med. 2020;383:1436-46.

2. Packer M, Anker SD, Butler J, Filippatos G, Pocock SJ, Carson $\mathrm{P}$, et al. Cardiovascular and renal outcomes with empagliflozin in heart failure. N Engl J Med. 2020;383:1413-24.

3. Guo W, Li M, Dong Y, Zhou H, Zhang Z, Tian C, et al. Diabetes is a risk factor for the progression and prognosis of COVID-19. Diabetes Metab Res Rev. 2020. https://doi.org/10.1002/dmrr.3319.

4. Hink U, Li H, Mollnau H, Oelze M, Matheis E, Hartmann M, et al. Mechanisms underlying endothelial dysfunction in diabetes mellitus. Circ Res. 2001;88:E14-22.

5. Koufakis T, Mustafa OG, Ajjan RA, Garcia-Moll X, Zebekakis P, Dimitriadis G, et al. The use of sodium-glucose co-transporter 2 inhibitors in the inpatient setting: is the risk worth taking? J Clin Pharm Ther. 2020;45:883-91.

6. Packer M. Do sodium-glucose co-transporter 2 inhibitors prevent heart failure with a preserved ejectionfraction by counterbalancing the effects of leptin? A novel hypothesis. Diabetes Obes Metab. 2018;20:1361-6.

7. Das L, Dutta P. SGLT2 inhibition and COVID-19: The road not taken. Eur J Clin Invest. 2020;50:e13339.

8. Tajbakhsh A, Gheibi Hayat SM, Akbari A, Inabadi M, Savardashtaki A, et al. COVID-19 and cardiac injury: clinical manifestations, biomarkers, mechanisms, diagnosis, treatment, and follow up. Expert Rev Anti Infect Ther. 2020. https://doi. org/10.1080/14787210.2020.1822737.

9. Dherange P, Lang J, Qian P, Oberfeld B, Sauer WH, Koplan B, et al. Arrhythmias and COVID-19: a review. JACC Clin Electrophysiol. 2020;6:1193-204.

10. Chen HY, Huang JY, Siao WZ, Jong GP. The association between SGLT2 inhibitors and new-onset arrhythmias: a nationwide population-based longitudinal cohort study. Cardiovasc Diabetol. 2020;19:73.

11. Zelniker TA, Bonaca MP, Furtado RHM, Mosenzon O, Kuder JF, Murphy SA, et al. Effect of dapagliflozin on atrial fibrillation in patients with type 2 Diabetes Mellitus: insights from the DECLARE-TIMI 58 trial. Circulation. 2020;141:1227-34.

12. Henry BM, Aggarwal G, Wong J, Benoit S, Vikse J, Plebani M, et al. Lactate dehydrogenase levels predict coronavirus disease 2019 (COVID-19) severity and mortality: a pooled analysis. Am J Emerg Med. 2020;38:1722-6.

13. Cure E, Cure MC. Can dapagliflozin have a protective effect against COVID-19 infection? A hypothesis. Diabet Metab Synd. 2020;14:405-6.

14. Oliveira CB, Lima CAD, Vajgel G, Campos Coelho AV, SandrinGarcia P. High burden of acute kidney injury in COVID-19 pandemic: systematic review and meta-analysis. J Clin Pathol. 2020. https://doi.org/10.1136/jclinpath-2020-207023.

15. Menon R, Otto EA, Sealfon R, Nair V, Wong AK, Theesfeld CL, et al. SARS-CoV-2 receptor networks in diabetic and COVID-19 associated kidney disease. Kidney Int. 2020;98:1502-18.

16. Heerspink HJL, Perco P, Mulder S, Leierer J, Hansen MK, Heinzel A, et al. Canagliflozin reduces inflammation and fibrosis biomarkers: a potential mechanism of action for beneficial effects of SGLT2 inhibitors in diabetic kidney disease. Diabetologia. 2019;62:1154-66.

17. Dalan R, Ang LW, Tan WYT, Fong SW, Tay WC, Chan YH, et al. The association of hypertension and diabetes pharmacotherapy with COVID-19 severity and immune signatures: an observational study. Eur Heart J Cardiovasc Pharmacother. 2020. https://doi. org/10.1093/ehjcvp/pvaa098.

18. Armeni E, Aziz U, Qamar S, Nasir S, Nethaji C, Negus R, et al. Protracted ketonaemia in hyperglycaemic emergencies in COVID19: a retrospective case series. Lancet Diabetes Endocrinol. 2020;8:660-3.

19. Chamorro-Pareja N, Parthasarathy S, Annam J, Hoffman J, Coyle C, Kishore P. Letter to the editor: unexpected high mortality in COVID-19 and diabetic ketoacidosis. Metabolism. 2020;110:154301.

20. Batista DV, Vieira CAFA, Costa TA, Lima EG. COVID-19-associated euglycemic diabetic ketoacidosis in a patient with type 2 diabetes on SGLT2 inhibitor: a case report. Diabetol Int. 2020. https://doi.org/10.1007/s13340-020-00473-3.

Publisher's Note Springer Nature remains neutral with regard to jurisdictional claims in published maps and institutional affiliations. 